\title{
Coping with a coroner's inquest: a psychiatrist's guide ${ }^{\dagger}$
}

\author{
Paul St John-Smith, Albert Michael \& Teifion Davies
}

\begin{abstract}
SUMMARY
During the period 2000-2004 the average annual suicide rate in England and Wales was 10.2 deaths per 100000 population over 10 years of age. About a quarter of those who take their own lives are in contact with mental health services in the year before their death. This means that an average in-patient, sector or community psychiatrist is likely to experience the death of at least one patient by suicide in most years. Suicides by patients cause considerable distress for the psychiatrist that is unlikely to resolve until after the coroner's hearing. This article discusses suicide prevention and provides guidance for psychiatrists on preparing for a coroner's inquest following a patient's death that may have been by suicide.
\end{abstract}

\section{DECLARATION OF INTEREST}

None.

In England and Wales, about 1\% of the population have suicidal ideation in any one week (Jenkins 1998) and about 140000 people attempt suicide each year (Samaritans 1998); 3-5\% of the population engage in self-harm at some time in their lives, and of these about $25 \%$ will repeat self-harm within 4 years and 3-7\% will eventually die by suicide (Soomro 2008).

During the period 2000-2004 there were on average 4960 suicides per year in England and Wales, i.e. 10.2 per 100000 population over 10 years of age. Of these, 1358 (27\%) were in contact with mental health services in the year before their death. Among the 1358, 19\% had been in contact with services in the $24 \mathrm{~h}$ before death, $49 \%$ in the week before, $14 \%$ were in-patients at the time of death and 14\% had left hospital in the previous 3 months. The post-discharge suicides clustered in the first week after leaving hospital, and 22\% occurred before the first follow-up appointment (Appleby 2006). For the death of each psychiatric patient there is a psychiatrist who faces the aftermath.

This article directly addresses psychiatrists and is written with reference to the National Health Service in England and Wales. However, much of the advice and information here is relevant to psychiatrists and clinicians working in other environments.

\section{Immediate responses}

Suicides by patients cause considerable distress, including symptoms of bereavement and feelings of professional threat in psychiatrists, irrespective of their grade. These are unlikely to resolve until after the coroner's hearing. To 'cope' means to face and deal with responsibilities.

On hearing about a possible suicide it is crucial that you read the relevant policies and procedures of your trust. This may include recommendations regarding informing other members of the team, conducting urgent multidisciplinary case reviews (Box 1) and instigating local confidential inquiries.

Policy may suggest contacting a colleague or a mentor for support and also the trust's legal advisor or your medical defence organisation in the event of a contentious situation.

Discussing the case with the patient's general practitioner (GP) might not only serve communication, but may also give you new information and help (re-)establish contact with the family. The family will be in need of support because of the effects of bereavement and shock at the manner of death. Their grief may encompass anger towards staff members that they feel should have prevented the suicide. Contacting the family may make the inquest less adversarial.

On notification of a suicide, it is important to record all the relevant information about the death in the patient's notes, including the date, time, place

\section{BOX 1 Benefits of multidisciplinary reviews}

- All the available information can be gathered, including details of involvement by different professionals; this can be invaluable in preparing a comprehensive report

- The appropriateness of the management plan can be assessed and lessons learned that improve the future management of suicide risk (Appleby 2001)

- It can be decided whether, how and by whom those suffering the aftermath of a suicide should be approached

- It can be decided how and by whom patients who knew the deceased should be informed and offered support

- Staff can engage in open discussion of the death, express feelings, relieve distress and support the clinicians involved

\section{ARTICLE}

Paul St John-Smith is a Consultant Psychiatrist in Hertfordshire, UK. His interests are evidence-based psychiatry, the philosophy of science, and psychiatric training. Albert Michael is a Consultant Psychiatrist at the West Suffolk Hospital. His interests include psychiatric training, affective disorders, psychopharmacology and evidence-based management. Teifion Davies is Senior Lecturer in Community Psychiatry and Director of Undergraduate Psychiatry Teaching at the Institute of Psychiatry, King's College London. His interests include promoting multidisciplinary teamwork in mental health.

Correspondence Albert Michael, Wedgwood House, West Suffolk Hospital, Bury St Edmunds IP33 20Z, UK. Email: Albert.Michael@smhp.nhs.uk

tFor a commentary on this article, addressing the situation in Scotland, see pp. 17-22, this issue. 
and mode of death and how the death was notified. Time, date and sign each entry. It is illegal to alter any existing records, for example by adding 'no suicidal ideas' to an entry dated a month previously, even if you believe this to be true. However, if you think in retrospect that previous records are inadequate, it is advisable to add your additional information at the end of the notes, dated with the day on which you are writing.

It is often worth photocopying the complete set of notes in case the originals go missing or cannot be accessed. If the file is voluminous, copy at least a selection of the most important parts, such as notes, letters, risk assessment forms, discharge summaries, the last written entry and details of the last face-toface contact.

A comprehensive review of the psychiatrist's role following a patient's suicide can be found in Hodelet (2001) and Campbell (2002).

\section{The coroner: notification, report and hearing}

\section{Notification}

Sometimes a request from the coroner's office for a report is the first indication you have that a patient has died. Knowledge about coroners and inquests would be useful in such situations.

In England and Wales, coroners are independent judicial officers who hold office under the Crown. Coroners are appointed for a district. They are either lawyers, doctors or both, and they follow legislation specific to coroners and inquests.

\section{Reporting a death}

All sudden, unexpected and unnatural deaths that occur in England and Wales must be reported to the coroner. These officials enquire into and seek to establish the medical cause of deaths reported to them. An inquest is usually opened to record that a death has occurred, to identify the dead person and to issue the documents required for the burial or cremation. The inquest itself is a fact-finding inquiry limited to establishing who has died, when and where the death occurred and the cause of death and how it arose. The registrar of deaths needs this information to register the death. The coroner may well have ordered a post-mortem examination and, if finding that the death was due to natural causes, will then not open an inquest. However, if death still appears to be from an unnatural cause, or the cause is still in doubt (e.g. more investigations need to be ordered), the coroner on opening the inquest will adjourn until all the investigations are completed.

\section{The hearing}

An inquest is not a trial. It is an inquiry into the facts surrounding a death: a hearing of the evidence. The Coroner's Rules 1984 prohibit the coroner from determining questions of civil or criminal liability on the part of any named people (www.kcl.ac.uk/depsta/law/research/coroners/ 1984ruleshtml). The inquest may, however, determine systemic failures. The coroner does have the power to investigate not just the main cause of death, but also any acts or omissions that directly led to the cause of death. Other court proceedings often follow an inquest, particularly a civil claim for damages. Where a person is to be charged with the murder or manslaughter of the deceased or causing their death by dangerous driving, the coroner is obliged to adjourn the inquest and will consider resuming it once those proceedings have been completed. The inquest may help the family of the deceased find out what happened. The information so obtained may be helpful in avoiding similar incidents in future.

Most inquests are held without a jury. A jury is involved if:

- the person died in prison

- the death was caused by an occurrence that needs to be reported to a government department

- the death occurred in circumstances that may affect the health or safety of the public, or

- the coroner decides that it is necessary to have a jury.

The coroner decides which witnesses to call and in which order they will give evidence. The coroner can also admit documentary evidence, either by reading a statement out in full or summarising it. If someone feels that they have relevant evidence they should contact the coroner as soon as possible after the death. If the coroner decides that the evidence is indeed relevant they will summon the individual to attend the inquest. Witnesses who receive a formal summons are obliged to attend. Witnesses who fail to attend when summoned are liable for a heavy fine and possibly punishment by way of imprisonment for contempt of court. A psychiatrist may be required to provide a written report and/or to attend and answer questions. Persons suspected of causing a death and required to give evidence at the inquest have the right not to answer questions that might incriminate them.

At the inquest, anyone who has a 'proper interest' may question witnesses, including professional or expert witnesses (Box 2). With the exception of the Chief of Police, 'properly interested people' may question the witnesses themselves or through lawyers. The questions must be sensible and relevant. This is something the coroner decides. Speeches are not allowed. If the patient was detained under the Mental Health Act, Mental Health Act commissioners may question the witnesses at the inquest. 
BOX 2 People with a 'proper interest' in an inquest

- The parents, spouse, children or anyone acting for the deceased

- Anyone who gains from a life insurance policy on the deceased

- Any insurer having issued such a policy

- Anyone whose actions the coroner believes may have contributed to the death, including employers or motorists causing a death

- The Chief Officer of Police Iwho may question witnesses only through a lawyerl

- Any person appointed by a government department to attend the inquest

- Anyone else the coroner decides has a proper interest

If the family involves lawyers, it would be wise to ask your trust to do the same.

The verdict

There are a number of possible verdicts that a coroner and jury can bring. They include: death by natural causes; accident; suicide; lawful or unlawful killing; industrial disease; and indeterminate cause, which is referred to as an open verdict. An open verdict is declared when there is insufficient evidence to determine the cause of death and how it arose. It is also possible to give a narrative verdict, which includes a short form of words encapsulating the major findings of facts. Coroners and juries often make findings as to the disputed facts that led to the conclusion of the death. This is particularly common in cases where death resulted from the actions of someone who was detained under the Mental Health Act.

If it appears to the coroner at the conclusion of the inquest that action is necessary to prevent a recurrence of situations similar to those occurring in the death in question, the coroner can draw attention to this publicly and announce that they will be notifying relevant authorities (e.g. the local council, government department or the mental health services) of the facts. This is often described as the coroner's recommendation, but it is in fact a report rather than a recommendation.

Inquests are held in public and consequently the press can be present. Every death is a personal tragedy, and coroners try to treat the survivors and witnesses sympathetically. Suicide notes and personal letters are not usually read out. Although every attempt is made to avoid making public the details of the private lives of the deceased person and their relatives, sometimes in the interests of justice it is unavoidable.

After the inquest, anyone who has a proper interest in the inquiry may apply to see the coroner's

notes or may have a copy of the notes on payment of a fee. Copies of tape-recordings or other digital recordings, if any, or transcripts of the hearing can also be obtained but the fee may be quite high.

\section{The report for the coroner}

You may be asked to provide a report for the coroner. A good report may reduce the likelihood of being summoned to give evidence. It will certainly assist the coroner in deciding the relevant information for the inquiry. It can be an opportunity to demonstrate the quality of the care plan and the care provided. The report should be comprehensive, covering the relevant history up to the time of death in chronological order (Box 3). It is often worth getting a colleague to check the report.

The letter of instruction from the coroner may include specific questions in addition to the request for the report. You should answer these point by point at the end of your report. Do not anticipate the verdict and do not express an opinion as to whether this was suicide or not. That is the responsibility of the coroner.

Be aware that the relatives and their solicitors might have access to these documents. Issues of confidentiality should be carefully considered. Nothing relevant should be omitted, but it would be better to be circumspect when mentioning other people's personal details.

\section{Going to court}

Attendance at an inquest can be stressful. We recommend that all psychiatrists sit in on one, as it can be a very informative experience. If you are summoned to attend an inquest, having a trial run with a colleague, especially one who has recently been to an inquest, will be helpful.

The venue of an inquest may vary from a small office to a formal courtroom. After you receive notification of the venue, it would be worth visiting it if this is possible, in order to assess the

\section{BOX 3 The report for the coroner}

This should include the following recent information:

- recent psychiatric history

- recent risk assessments

- recent mental state examinations

- care plans

- contacts and treatments

- a summary and/or a formulation and a diagnosis using ICD-10 criteria
It may also include background information:

- personal and family history

- drug and alcohol history

- cultural and spiritual identity

- personality

- past medical history

- past psychiatric history

- documentation such as discharge summaries, risk and psychological assessments, treatment plans (add lengthy documents as appendices) 
setting. Remember that coroners follow different procedures, for example some like witnesses to stand and others are happy for them to sit.

Review the patient's medical records immediately before the inquest, especially if there has been a long adjournment, and take them with you on the day. A pre-inquest meeting within the trust can help everyone involved be better prepared.

Dress professionally and smartly for the court. Arrive early, allowing adequate time for parking and so on. On arrival at the venue, report to the receptionist, who will give you a 'visitor' identity badge. Inform the coroner's officer about the sort of affirmation or oath you wish to take. Be prepared for a long wait, depending on what other cases are being heard that day. It can add to everyone's stress if you have to sit wait for long in the same room as anxious, grieving or angry relatives. You could ask for a separate waiting-room. It might help to take a colleague with you, both for distraction in the waiting-room and for support throughout the proceedings.

In addition to the relatives, who might also bring lawyers, a number of other people may be present at the inquest (and in the waiting-room). These include police officers, pathologists, the GP and other staff from your trust. There may be advocates from user and carer organisations such as Rethink or MIND to support the family. There may be expert witnesses hired by the family or support organisations. They may work with the lawyers for the family in a crossexamination. In high-profile cases it is advisable to seek advice from your trust's public relations officer and your medical defence organisation regarding possible questions from the press.

On your way out, return your identity badge and collect a fee claim form from the coroner's officer.

Calthorpe \& Choong (2004) provide a useful overview of the coroner's court and further information is available on the Department for Constitutional Affairs' website (www.dca.gov.uk/ corbur/coronfrhtm).

\section{Prediction and screening}

\section{Prediction}

During the inquest, you may be subjected to adversarial questioning by the family or experts and lawyers representing them. Up-to-date information on suicide, risk assessment and risk management, in both the hospital and the community, will be essential in responding to questioning and informing the coroner. It is also necessary for assisting your trust's internal inquiry and, most importantly, when meeting the bereaved. Such information (e.g. Gunnell 1994; Appleby 1999, 2001, 2006) is readily accessible to the public and the bereaved, let alone lawyers and other expert witnesses.

If cross-examined you may be forced to defend your inability to predict suicides. This necessitates explaining with empathy the genuine problem of accurate empirical prediction and prevention of suicides in clinical practice. The lawyers for the deceased may quote scales for measuring and predicting suicides that 'you did not make the best use of'. You should be able to explain how their levels of sensitivity, specificity and positive predictive value make such scales of very little use in predicting immediate and short-term suicide risk.

\section{About risk factors}

To identify risk factors for suicide in psychiatric inpatients and to evaluate their power in detecting people at risk of suicide, Powell and colleagues compared 112 people who took their own lives while in-patients in psychiatric hospitals with 112 randomly selected control patients in the same hospitals (Powell 2000). They calculated an estimated suicide rate for psychiatric in-patients of 13.7 (95\% CI 11.7-16.1) per 10000 admissions, and found five strongly predictive factors (adjusted likelihood ratio LR >2). These were:

- actual suicide attempt $(\mathrm{LR}=4.9)$

- family history of suicide ( $L R=4.6)$

- planned suicide attempt $(\mathrm{LR}=4.1)$

- recent bereavement $(L R=4.0)$

- presence of delusion ( $\mathrm{LR}=2.3$ )

- chronic mental illness (LR=2.2).

Only 2 of the 112 patients who died by suicide had a predicted risk above 5\%. The authors concluded that even in this high-risk group the clinical utility of these risk factors is limited because of their low sensitivity and low specificity. With the rarity of in-patient suicide, even in this high-risk group, the positive predictive value of these factors is low and the number of false positives would be more than 99\%.

\section{Screening}

Understanding the sensitivity, specificity and positive predictive value of screening tools and their applicability in different situations is essential. Potential problems of screening are well described by Warner (2004).

The US Preventative Services Task Force (2004) found only limited evidence that the available screening tools, including tools to identify those at high risk, accurately identified people at risk of suicide in primary care settings. They found no or insufficient evidence that screening or treating those at high risk reduced suicide attempts or mortality. 
The known risk factors for suicide include male gender, suicidal ideation on admission, diagnosis, outcome on discharge and the number of previous suicide attempts. Goldstein and colleagues assessed these risk factors and recorded suicides over a 10year period in 1906 patients with affective disorder admitted to a psychiatric hospital (Goldstein 1991). They were unable to identify with a $50 \%$ probability any one of the 46 patients who died by suicide during this period. They could identify only one of them with a $15 \%$ probability.

Previous child and adolescent psychiatry patients are known to have higher risk of suicide. One study (Engqvist 2006) followed up 1400 such individuals for between 12 and 33 years. There were 38 deaths, 34 of which were by suicide. However, only 2 of these 34 people had been admitted for attempted suicide.

\section{A practical example: the Manchester Self-Harm Rule}

The Manchester Self-Harm Rule was developed from the study of over 9000 patients who attended emergency departments with self-harm (Cooper 2006). The instrument, for assessing suicide after self-harm, uses four clinical correlates: any history of self-harm; previous psychiatric treatment; benzodiazepine use in the current attempt; and any current psychiatric treatment. The study reported a sensitivity of $94 \%$ and a specificity of $25 \%$ in identifying high-risk patients. Of those identified, $17 \%$ re-attended and 22 died by suicide within 6 months.

Let us apply this instrument to screen a population of 100000 people with severe mental illness and therefore an annual suicide rate 30 times the general population rate, i.e. 300/100000. Table 1 shows the figures and the numbers of false positives and false negatives given the reported sensitivity (94\%) and specificity (25\%) of the instrument.

Among this group of 100000 people, 300 will die by suicide within a year and 99700 will not. With the 25\% specificity of the Manchester Self-Harm Rule we will correctly identify 24925 as not at risk of suicide. We will also identify $75 \%$ of the 99700 , i.e. 74775 , as at risk of suicide when they are not.

Among the 300 who will die by suicide, the instrument will identify 94\%, i.e. 282. However, it will not identify $6 \%$ (18) of them.

The positive predictive value, i.e. the chance of having the condition in question given a positive test result, is calculated as $\mathrm{PPV}=a / a+b$. Thus, from Table 1 the Manchester Self-Harm Rule in this example has a PPV of $0.38 \%$. Its negative predictive value, i.e. the chance of not having the condition in question given a negative test result, is $\mathrm{NPV}=d / d+c=99.9 \%$.
TABLE 1 Suicide prediction in a population $(n=100000)$ of people with severe mental illness

\begin{tabular}{l|c|c|c}
\hline & Suicide risk present & Suicide risk absent & Total \\
\hline \multirow{2}{*}{$\begin{array}{l}\text { Screening test } \\
\text { positive }\end{array}$} & $a$ & $b$ & $a+b$ \\
& True positive & False positive & 75057 \\
\hline \multirow{2}{*}{$\begin{array}{l}\text { Screening test } \\
\text { negative }\end{array}$} & 282 & 74775 & $c+d$ \\
& False negative & True negative & 24943 \\
\hline \multirow{2}{*}{ Total } & 18 & 24925 & $a+b+c+d$ \\
& $a+c$ & $b+d$ & 100000 \\
\hline
\end{tabular}

Thus, this instrument will accurately identify non-suicidal patients 99\% of the time. However, compared with 75057 people not ruled out as not at risk of suicide, only 282 would really be at risk of suicide.

If we assume that hospital admission would prevent all suicides, one would have to admit 75057 people in order to prevent 282 suicides and would still miss 18 suicides.

\section{Causation}

Causation is a central topic in the philosophy of science. We attempt to use science and controlled studies to liberate us from our biased misconceptions about causes. Yet if one considers the MMR vaccine autism scare, public misconception may be fuelled by ill-advised reporting of research. Moreover, generalisation of causes from epidemiological and/ or group studies to the individual or vice versa is a problem for attributing or predicting causation. Both legal and medical approaches to causation emphasise integrity of the process of gathering and presenting information. Evidence then needs to be critically appraised according to the findings. There are rules of evidence and standards of certainty that should be used in both procedures. Epidemiological and research evidence (including that from the National Institute for Health and Clinical Excellence (NICE) and the National Confidential Inquiry into Suicide and Homicide by People with Mental Illness), as well as individual case history presented should be viewed in this light. Appropriate safeguards against rash opinions as distinct from an adequate investigatory process also are needed. It is important that after a coroner's hearing 'justice' is done and seen to be done.

The National Patient Safety Agency's (NPSA's) 'seven steps to patient safety' (Box 4) may be used as a model after serious untoward incidents. The NPSA admits that there is no single way to measure patient safety. However, paradoxically, an increase in reporting of patient safety incidents is a sign that organisations have implemented an open and fair culture in which staff learn from things that go wrong. For example, in the aviation industry, as 
BOX 4 Seven steps to patient safety

1 Build a safety culture

2 Lead and support the staff

3 Integrate risk management activity

4 Promote reporting

5 Involve and communicate with patients and the public

6 Learn and share safety lessons

7 Implement solutions to prevent harm

INational Patient Safety Agency 2004

reporting goes up, the number of serious incidents begins to decline (Miyagi 2005).

\section{Root cause analysis and barrier analysis}

Step 6 of the NPSA guidance, 'learn and share safety lessons', involves encouraging staff to use root cause analysis (Boxes 5,6) to learn how and why incidents happen.

Organisational and strategic factors can contribute to untoward incidents both directly and indirectly, through contextual and local environmental factors and by allowing the breakdown of preventive and protective measures, resulting in human errors. However, the findings of root cause analysis are processed higher up in organisations, and elements other than the 'weakest link', i.e. the most junior or the least qualified member of staff involved in the chain of events, may not be considered as needing remedial action. Ideally, management should bear responsibility for staffing and allocation of resources.

Barrier analysis is another investigational method that involves tracing potential pathways by which the patient might have been adversely affected, including the identification of any failed or missing measures that might have prevented the undesired outcome. It is conceptually simple, requires minimal resources, works well in combination with other measures and can translate naturally into corrective action (National Patient Safety Agency 2004).

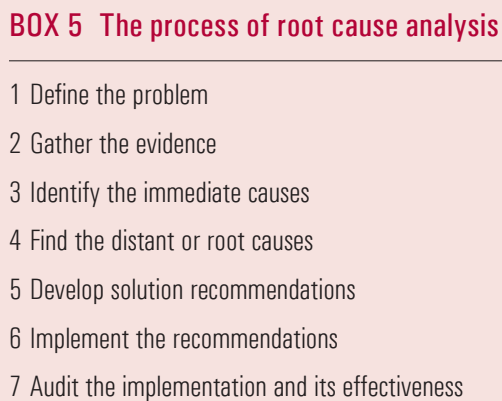

However, in our opinion it is often overly subjective, promotes simple deterministic thinking, can confuse cause with effect or antecedent, and the reliability is frequently low in complex, abstract and nondeterministic areas such as prediction in psychiatry. In medicine, and in epidemiology in particular, the Bradford-Hill criteria (Box 7) are the cornerstone of establishing causation, but they rely on series, cohorts and so on, not individual instances of failure (Bradford-Hill 1965).

There is always a potential for error and bias when evaluating single cases retrospectively. This arises in part from finding apparent or erroneous causes that affirm the consequent (e.g. the patient was prescribed an antipsychotic drug, therefore he had a psychotic disorder) or are incorrectly attributed because of post hoc, ergo propter hoc reasoning (e.g. the patient died by suicide while on leave, therefore allowing her leave caused her to take her life). Both are formal fallacies. Another example of such reasoning is the assumption that if someone dies by suicide they must have been mentally ill and therefore their death is a failure of psychiatric care. This will be true in some cases, but not all. Other sources of individual case bias include biases of confirmation, mechanism and interpretation.

\section{Strategies for prevention}

\section{Suicide by hanging}

Meehan and colleagues studied 4859 suicides among people who had been in recent contact with mental health services in England and Wales between 1996 and 2000 (Meehan 2006). Of these, 754 (16\%) were in-patients at the time of death. Nearly a quarter of these suicides occurred within the first 7 days of admission and 236 (31\%) occurred on the ward, the majority by hanging. A further 1100 (23\%) died within 3 months of discharge from in-patient care. Post-discharge suicides occurred most frequently within the first 2 weeks after leaving hospital, and the highest number occurred on the first day. The team concluded that improving ward design and removing fixtures that could be used in hanging might reduce suicides among in-patients. To prevent post-discharge suicides they recommended early community follow-up after discharge and closer supervision of high-risk patients. However, there is no evidence on the effectiveness of these measures.

Gunnell and colleagues reviewed the literature on hanging since 1966 (Gunnell 2005a). They found that only $10 \%$ of suicides by hanging occur in controlled environments such as hospitals, prisons or police custody; the majority occur in the community, where ligatures and ligature points are widely available. Although hanging is fatal in about 70\% 
BOX 6 Common elements of root cause analysis

- Materials, e.g. the type of drug/treatments available

- Equipment, e.g. poor maintenance

- Environment, e.g. poor lay out of work place, architecture of ward

- Management, e.g. tasks not supervised properly, excess demands, dysfunctional teams

- Methods, e.g. poor procedures, practices and communication/ human errors

- Management systems, e.g. poor training or education, previous recommendations not implemented

of cases, $80-90 \%$ of those who reach hospital alive survive. They concluded that prevention strategies focused on restriction of access to means of hanging are of limited value. Strategies to reduce suicide by hanging should focus on prevention in controlled environments, the emergency management of 'nearhanging' and the primary prevention of suicide in general.

\section{Department of Health recommendations}

The first report on the National Confidential Inquiry into Suicide and Homicide by People with Mental Illness (Appleby 1999) made a number of recommendations, among which those listed in Box 8 formed the basis of the National Suicide Prevention Strategy for England (Department of Health 2002).

There is no evidence for the efficacy of these measures in reducing suicides. Some of these points are outside the remit of the psychiatrist but within the responsibility of the trust. However, it will be useful to know these action points when you appear before the court. You are seen, perhaps unfairly, as representing your trust in a coroner's court and may be questioned on ward design, and health and safety strategy and reviews.

\section{BOX 7 The Bradford-Hill criteria for causation}

- Strength (statistical

- Consistency of findings (reproducibility)

- Temporal sequence (order of events)

- Biological gradient (dose-response relationship)

- Specificity (the cause leads to a unique outcome)

- Coherence /consistency with existing knowledge

- Biological plausibility (psychiatric validity)

- Reasoning by analogy (from similar models)

- Experimental evidence (prospective)

\section{Medication and suicidality}

You may be questioned on the effect of medication on suicidality, i.e. suicidal thoughts and behaviour. Both lithium (Burgess 2001) and clozapine (Meltzer 2002; Hennen 2005) may have an antisuicidal effect. However, this effect may be related to the regular and frequent monitoring required for these drugs rather than their direct pharmacological action. Although selective serotonin reuptake inhibitors (SSRIs) and atypical antipsychotics are less likely than older drugs to cause death by suicide, especially in overdose, this is not the same as reducing suicidality. There have been suggestions that the SSRIs may actually increase suicidality, possibly by causing akathisia (Fergusson 2005; Gunnell 2005b). However, akathisia also occurs in $20-75 \%$ of patients taking antipsychotic drugs (King 2004), without necessarily increasing the risk of suicide.

In England during 1997-2004 there was a fall in the number of deaths from self-poisoning with tricyclic antidepressants. However, the number of suicides among patients with depression did not fall. Presumably they used some other means (Morgan 2004).

\section{Hospital admission}

For patients in crisis, it is generally assumed that admission to hospital or containment at home in the continuous care of home treatment or assertive outreach teams will reduce suicide rates. However, there have been no prospective randomised placebo-controlled studies of the efficacy of any

\section{BOX 8 Strategies for suicide prevention}

- Regular (every 3 years) staff training in risk management

- In-patients with severe mental illness and a history of self-harm or violence should receive the highest level of care under the care programme approach

- Individual care plans should specify action to be taken if a patient fails to adhere to treatment or to attend appointments

- Prompt access to services for people in crisis and their families

- Assertive outreach teams to prevent loss of contact with vulnerable and high-risk patients

- Atypical antipsychotic drugs to be available for all patients with severe mental illness prescribed typicals who are non-adherent because of drug side-effects
- Local strategies for dual diagnosis that include training in the management of substance misuse services and employment of staff with specific responsibility for developing the local service

- Removal or covering of all likely ligature points in in-patient wards

- Follow-up within 7 days of hospital discharge for everyone with severe mental illness or a history of self-harm in the previous 3 months

- Patients with a history of self-harm in the previous 3 months to receive supplies of medication covering no more than 2 weeks

- Local arrangements for information-sharing with criminal justice agencies

- Policy ensuring post-incident multidisciplinary case review and provision of information to the patient's family

(Department of Health, 2002) 
of these measures and there is no evidence that temporary hospital or home containment ultimately reduces suicide rates among people thus treated. Nevertheless, if mental illness is making someone particularly suicidal or impulsive, containment with high levels of supervision may, in the short term, prevent suicide. It is good clinical practice to monitor patients closely when they are particularly at risk, disturbed or impulsive, although it should be noted that some individuals have personalities that make them enduringly impulsive, and their agitation and impulsivity may be worsened if they are under close observation. The assumption that the level of observation of people with acute mental illness can be reduced when symptoms improve is not evidence based: only hindsight can decide on the right or wrong of a decision to alter observation levels.

As mentioned above (Appleby 2006), during 2000-2004, 856 deaths by suicide, i.e. 14\% of all suicides by people with mental illness, occurred while the individuals were in-patients. Of these, $81 \%$ were assessed to be at no or low immediate risk of suicide and $49 \%$ were considered to be at low or no long-term risk of suicide; $22 \%$ of those who died on the ward were under special observation. A further $14 \%$ of suicides by people with mental illness occurred within 3 months of discharge from an in-patient unit. Among these, 15\% occurred within a week and $22 \%$ before the first follow-up appointment. The report noted that suicides by inpatients fell from $17 \%$ of all suicides by people with mental illness in 1997 to only 11\% in 2004 (i.e. 67 fewer deaths), but this may simply be secondary to the reduction in psychiatric bed numbers.

\section{Preventable suicides}

In their second report on the findings of the National Confidential Inquiry (Appleby 2006), the authors tried to identify the preventable suicides, i.e. those who died in close proximity to services, especially those who were at evident risk but did

\section{BOX 9 Measures to reduce suicides}

- Reduce absconding from in-patient units

- Strengthen the transition from ward to community

- Ensure that high-risk patients receive enhanced CPA, backed by peer review in the highest-risk cases

- Respond robustly when care plans break down

- Accept that prevention is possible in many cases particularly in in-patient suicides not receive appropriate support. They highlighted three groups:

- 349 in-patients who took their lives while under close observation, within 7 days of admission, on home leave but living alone, or on absconding from the ward $(41 \%$ of the 856 in-patient suicides)

- 255 patients who took their lives before their first follow-up ( $22 \%$ of the post-discharge suicides)

- 436 patients who were not on the enhanced care programme approach (CPA) following informal admission, or despite severe mental illness or recent self-harm, and 68 patients who, although on CPA, were non-adherent and/or missed appointments (12\% of community suicides).

Thus, with hindsight, 1078 (22\% of the 4942 suicides in England and Wales between April 2000 and December 2004) might be considered preventable. This means that they did not consider preventable the remaining $78 \%$ of suicides by people with possible mental health problems, which formed only $27 \%$ of all suicides.

Even though $49 \%$ of the patients who died by suicide had been in contact with services in the previous week, at the final contact immediate suicide risk was rated to be low or absent in $86 \%$ of cases. Moreover, among the 1271 people who died by suicide within 3 months of discharge from in-patient care, there was no clear change in risk associated with the post-discharge period.

It should be noted that the report's authors depended on the opinion of the psychiatrists involved in the care of the deceased. One cannot ignore the fact that there was no evidence base or scientific rigour to the process by which preventability was decided. It does not seem unlikely that the question regarding preventability tapped more into the frustration of the psychiatrist than into the empirical facts of the death.

The report noted that there has been a rise (proportionate or concomitant?) in suicides by patients outside the priority groups. Thus, when suicides in one area or by one means are reduced, they seem to increase in other areas or by other means.

Masterton \& Cavanagh (2004) summarised the problems in preventing suicide. Suicide is a rare and unpredictable event even among those who are at high risk. Suicidal intent is not constant. It waxes and wanes, often suddenly and unexpectedly. Moreover, the risk can be greater when the patient is becoming ill, is recovering or has recovered, than when severely ill.

The report's recommendations (Box 9) mostly represent pearls of good clinical practice and common sense, albeit not systematically evidence based. 
The National Confidential Inquiry uses the Suicide Questionnaire to enable systematic exploration of the facts in cases of suicide (National Confidential Inquiry into Suicide and Homicide by People with Mental Illness 2005). It is sent out to consultants of every patient who dies by suicide. It is worth looking at a copy, before you are faced with it. In our experience the questionnaire does not go into the distant root causes, which are unique to each case.

\section{Surprises}

As discussed earlier, a number of different verdicts are available to the coroner, and deaths that initially appear to have been suicide may not be recorded as such. To bring a verdict of suicide, the coroner must be satisfied beyond reasonable doubt that the deceased did the act that caused their death, that they knew that it would cause their death and that they really wanted to die. If the coroner believes that the act that caused a death was in fact a 'cry for help', they will often bring in a verdict of accidental death, because death was the unwanted consequence of the individual's action. If, on the balance of possibilities, the coroner cannot be satisfied that it was a cry for help but cannot be sure that the individual really wanted to kill themselves an open verdict may be brought in.

Sometimes mental health teams greatly distressed by the apparent suicide of a patient find at the inquest that an unsuspected physical disease caused the death. Moreover, certain physical events can cause accidents that look like suicide. Deaths are more likely to be due to natural causes than to suicide even in people with mental illnesses. It is therefore worth waiting for all the information, including the post-mortem and toxicology reports and the outcome of the inquest. There is one simple, though rarely used, measure that trusts can take to reduce everybody's distress and the chance of surprises: they can ask the coroner's office for a copy of the post-mortem and toxicology reports. At present, there is a statutory fee of $£ 1.10$ per page.

On occasions adversarial parties demand explanations of a psychiatrist's failure to predict and prevent a suicide, claiming that suicide prevention 'is not rocket science'. One might agree with this assertion: suicide prevention is much more complicated and far less predictable. Human beings are not inert objects whose behaviour is simply predicted by Newtonian physics. People are able to deny or mislead others regarding their intentions, which complicates predictions.

In the aftermath of a verdict consider a review with the multidisciplinary team to look at the impact the case has had and whether further contact needs to be made with the family.

\section{Conclusions}

As no suicide-prevention intervention has been demonstrated to be effective in well-conducted clinical trials, Masterton \& Cavanagh assert that the belief that medical interventions could prevent suicide not only has no evidence base but runs the risk of raising false expectations among the public and of encouraging grieving relatives to blame healthcare professionals inappropriately (Masterton 2004). Nevertheless, there is no excuse for blatantly poor clinical practice, and the inquiry process may reveal grossly negligent or dangerous practice in individual cases.

Looking at suicide prevention another way, one could argue that, to guarantee the prevention of a particular suicide, a health worker would not only have to successfully predict the if, when and where factors for the individual, but also have an effective and permanent solution or treatment. Again, not having $100 \%$ effective predictions or treatments is no excuse for not trying. Predicting suicide is like predicting fatal road accidents. It is probabilistic, not deterministic. Dangerous behaviours do not always result in death; conversely, deaths occur in people who are at no obvious increased risk. The fact that finite but evidence-based predictors and prevention strategies do not offer 100\% certainty should not stop us from using them in both cases.

Suicide is epidemiologically relatively rare but clinically quite common. Given the number of patients at high risk of suicide, it is inevitable that sooner or later some of these individuals will die by suicide. It is therefore important that clinicians keep up to date with suicide risk assessment and prevention strategies, the difficulties of predicting and preventing suicides, and their own and their trust's responsibilities in relation to their patients.

\section{Acknowledgements}

We thank Edward G. Thomas, Her Majesty's Coroner for the district of Hertfordshire and Dr Lester Sireling, Consultant Psychiatrist in Barnet, for their comments.

\section{References}

Appleby L, Sherratt J (2001) Good clinical practice on suicide and suicide prevention. Psychiatric Bulletin; 25: 41-2.

Appleby, L, Shaw, J, Amos, T, et al (1999) Safer Services: Report of the National Confidential Inquiry into Suicide and Homicide by People with Mental IIIness. TSO (The Stationery Office).

Appleby L, Shaw J, Kapur N, et al (2006) Avoidable Deaths: Report of the National Confidential Inquiry into Suicide and Homicide by People with Mental IIIness. TSO (The Stationery Office).

Bayney R, St John-Smith P, Conhye A (2002) MIDAS: a new service for the mentally ill with comorbid drug and alcohol misuse. Psychiatric Bulletin; 26: 251-4.

Bradford-Hill A (1965) The environment and disease: association or causation? Proceedings of the Royal Society of Medicine; 58: 293-300. 


\begin{tabular}{lllll}
\multicolumn{5}{c}{ MCQ answers } \\
$\begin{array}{lllll}1 & 2 & 3 & 4 & 5 \\
\text { at } & \text { af } & \text { af } & \text { af } & \text { af } \\
\text { bf } & \text { bf } & \text { bt } & \text { bf } & \text { bt } \\
\text { cf } & \text { ct } & \text { cf } & \text { cf } & \text { cf } \\
\text { df } & \text { df } & \text { df } & \text { dt } & \text { df } \\
\text { ef } & \text { ef } & \text { ef } & \text { ef } & \text { ef }\end{array}$
\end{tabular}

Burgess S, Geddes J, Hawton, K, et al (2001) Lithium for maintenance treatment of mood disorders. Cochrane Database of Systematic Reviews; issue 3.

Calthorpe B, Choong S (2004) The coroner's court and the psychiatrist. Advances in Psychiatric Treatment; 10: 146-52.

Campbell C, Fahy T (2002) The role of the doctor when a patient commits suicide. Psychiatric Bulletin; 26: 44-9.

Cooper J, Kapur N, Dunning J, et al (2006) A clinical tool for assessing risk after self-harm. Annals of Emergency Medicine; 48: 459-66.

Department of Health (2002) National Suicide Prevention Strategy for England. Department of Health.

Engqvist U, Rydelius PA (2006) Death and suicide among child and adolescent psychiatric patients. BMC Psychiatry; 6: 51

Fergusson D, Doucette S, Glass KC, et al (2005) Association between suicide attempts and selective serotonin reuptake inhibitors: systematic review of randomised controlled trials. BMJ; 330: 396-9.

Goldstein RB, Black DW, Nasrallah A, et al (1991) The prediction of suicide. Sensitivity, specificity, and predictive value of a multivariate model applied to suicide among 1906 patients with affective disorders. Archives of General Psychiatry; 48: $418-22$

Gunnell D, Frankel S (1994) Prevention of suicide: aspirations and evidence. BMJ; 308: 1227-33.

Gunnell D, Bennewith 0, Hawton K, et al (2005a) The epidemiology and prevention of suicide by hanging: a systematic review. International Journal of Epidemiology; 34: 433-42.

Gunnell D, Saperia J, Ashby D (2005b) Selective serotonin reuptake inhibitors (SSRIs) and suicide in adults: meta-analysis of drug company data from placebo controlled, randomised controlled trials submitted to the MHRA's safety review. $B M J ; 330: 385-8$

Hennen J, Baldessarini RJ (2005) Suicidal risk during treatment with clozapine: a meta-analysis. Schizophrenia Research; 73: 139-45.

Hodelet N, Hughson M (2001) What to do when a patient commits suicide. Psychiatric Bulletin; 25: 43-5.

Jenkins R, Bebbington P, Brugha, TS, et al (1998) British psychiatric morbidity survey. British Journal of Psychiatry; 173: 4-7.
King DJ \& Waddington JL (2004) Antipsychotic drugs and the treatment of schizophrenia. In Seminars in Clinical Psychopharmacology (2nd edn) (ed DJ King): 316-80. Gaskell.

Masterton G, Cavanagh J (2004) Suicide and deliberate self-harm. In Companion to Psychiatric Studies (7th edn) (eds EC Johnstone, DG Cunningham Owens, SM Lawrie, et all: 661-82. Churchill Livingstone.

Meehan J, Kapur N, Hunt IM, et al (2006) Suicide in mental health in-patients and within 3 months of discharge. National clinical survey. British Journal of Psychiatry; 188: $129-34$.

Meltzer HY (2002) Suicidality in schizophrenia: a review of the evidence for risk factors and treatment options. Current Psychiatry Reports; 4: 279-83.

Miyagi M (2005) Serious Accidents and Human Factors: Aviation Safety through Incident Reporting Analysis. Wiley-Blackwell.

Morgan OWC, Griffiths C, Majeed A (2004) Association between mortality from suicide in England and antidepressant prescribing: an ecological study. BMC Public Health; 4: 63

National Confidential Inquiry into Suicide and Homicide by People with Mental IIIness (2005) Suicide Questionnaire. Version: 04/2005. NCIS Ihttp://www.medicine. manchester.ac.uk/psychiatry/research/suicide/prevention/nci/methodology/sample suicidequestionnaire.pdf).

National Patient Safety Agency (2004) Seven Steps to Patient Safety - Your Guide to Safer Patient Care. NPSA (http://www.npsa.nhs.uk/nrls/improvingpatientsafety/ patient-safety-tools-and-guidance/7steps)

Powell J, Geddes J, Hawton K, et al (2000) Suicide in psychiatric hospital inpatients. Risk factors and their predictive power. British Journal of Psychiatry; 176: 266-72.

Samaritans (1998) Exploring the Taboo. Samaritans.

Soomro GM (2008) Deliberate self-harm (and attempted suicide). BMJ Clinical Evidence; online 01:1012 http://clinicalevidence.bmi.com/ceweb/conditions/meh/ 1012/1012.jsp.

US Preventative Services Task Force (2004) Screening for suicide risk: recommendation and rationale. Annals of Internal Medicine; 140: 820-1.

Warner, J (2004) Clinicians' guide to evaluating diagnostic and screening tests in psychiatry. Advances in Psychiatric Treatment; 10: 446-54.

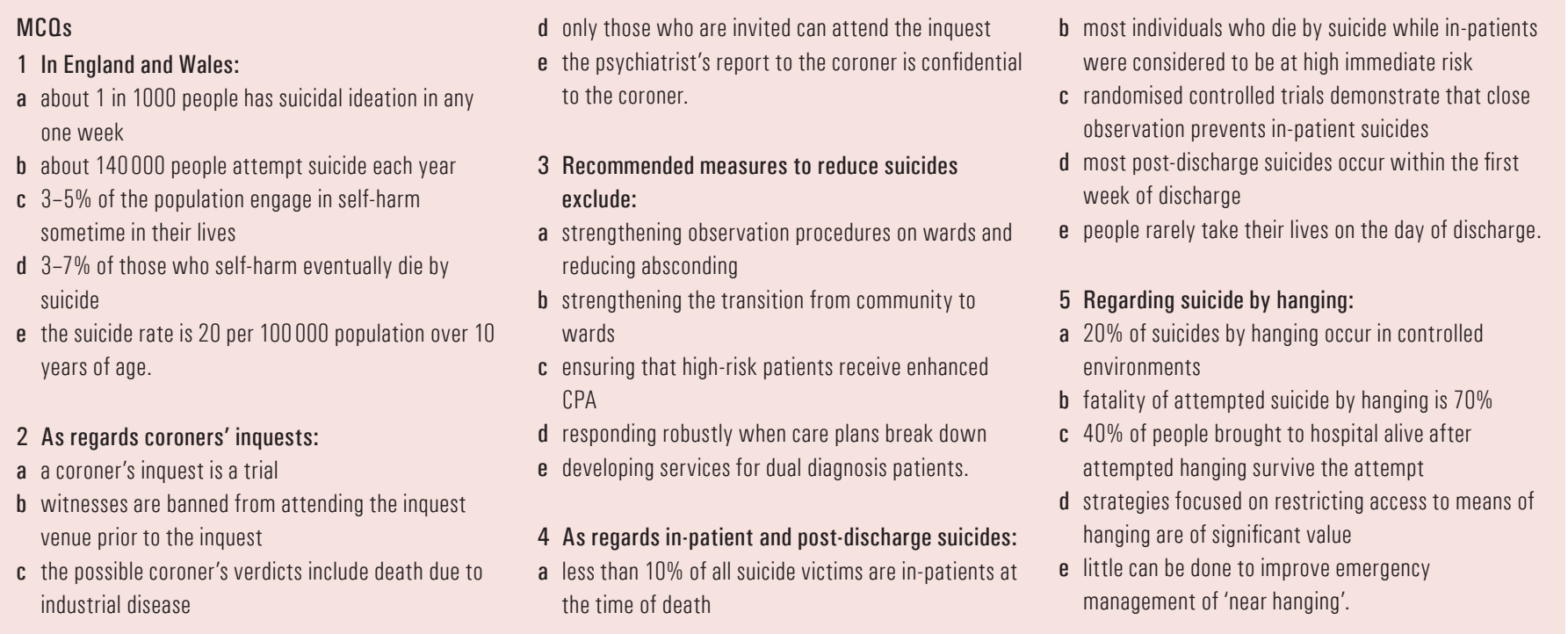

